Article

\title{
An Application of the Sonine-Letnikov Fractional Derivative for the Radial Schrödinger Equation
}

\author{
Okkes Ozturk $^{1, *}$ and Resat Yilmazer ${ }^{2}$ \\ 1 Department of Mathematics, Bitlis Eren University, Bitlis 13000, Turkey \\ 2 Department of Mathematics, Firat University, Elazig 23119, Turkey; rstyilmazer@gmail.com \\ * Correspondence: oozturk27@gmail.com
}

Received: 22 March 2019; Accepted: 3 April 2019; Published: 4 April 2019

\begin{abstract}
The Sonine-Letnikov fractional derivative provides the generalized Leibniz rule and, some singular differential equations with integer order can be transformed into the fractional differential equations. The solutions of these equations obtained by some transformations have the fractional forms, and these forms can be obtained as the explicit solutions of these singular equations by using the fractional calculus definitions of Riemann-Liouville, Grünwald-Letnikov, Caputo, etc. Explicit solutions of the Schrödinger equation have an important position in quantum mechanics due to the fact that the wave function includes all essential information for the exact definition of a physical system. In this paper, our aim is to obtain fractional solutions of the radial Schrödinger equation which is a singular differential equation with second-order, via the Sonine-Letnikov fractional derivative.
\end{abstract}

Keywords: fractional calculus; Sonine-Letnikov fractional derivative; generalized Leibniz rule; radial Schrödinger equation

\section{Introduction}

The derivative concept with fractional order was first mentioned by L'Hospital in a letter sent to Leibniz in 1695. Since then, it has been an interesting work item and, many theories on this subject have been developed and are still being developed. Some applications of fractional calculus can be seen in recent studies such as fractional differential equations [1], fractional subdiffusion equations [2], non-Gaussian distributions to random walk in the context of memory kernels [3], response functions [4] and fractional Prabhakar derivative [5].

The generalization of the Cauchy differentiation formula to non-integer orders, which has been suggested by Sonine [6] in 1872, has an important place in the fractional calculus. This generalization has been adjusted by Letnikov [7] in 1873. Therefore, this formula, which forms the basis of the generalized Leibniz rule, may be called the Sonine-Letnikov fractional derivative (see reference [8]). In some studies, the generalized Leibniz rule has been called the Nishimoto fractional derivative (N-fractional calculus operator) instead of the Sonine-Letnikov fractional derivative because Nishomoto has also studied on the generalization of the Cauchy differentiation formula since 1976 (see references [9-11]). However, the basis of this rule has been mentioned by Sonine. It is possible to see many works based on the Sonine-Letnikov fractional derivative, although it is often known as $\mathrm{N}$-fractional calculus operator. These works include the solutions of the Gauss equation [12], solutions of modified Whittaker equations [13], an almost free damping vibration equation [14], differential operators and integral operators in univalent function theory [15], geometric univalent function theory [16], power and logarithmic functions, Weber's equation, Gauss hypergeometric equations and some double infinite, finite and mixed sums [17], products of some power functions and some doubly infinite sums [18], some composite functions [19], some algebraic functions [20], some functions which include a root sign [21], a modified hydrogen atom equation [22], some second order homogeneous 
Euler's equation [23], some logarithmic functions and some identities [24], fractional solutions of homogeneous and nonhomogeneous Chebyshev's equations [25,26], explicit solutions of Gegenbauer equation [27], fractional solutions of Bessel equation [28], fractional solutions of the radial part in the fractional Schrödinger equation [29] and some singular differential equations [30].

The fractional quantum mechanics and fractional Schrödinger equations were first considered by Vladimirov et al. [31]. The fractional calculus studies have been also discussed in the different fields of fractional quantum theory such as space-fractional Schrödinger equation [32], fractional Heisenberg equation [33], quantization of fractional derivatives [34], fractional generalization of the quantum Markovian master equation [35], fractional dynamics of open quantum systems [36], quantum dissipation from power-law memory [37], fractional quantum field theory [38], time-fractional Schrödinger equation [39] and time-dependent fractional dynamics with memory in quantum physics [40]. In addition to all these, we intended to use a Sonine-Letnikov fractional derivative for the radial Schrödinger equation in this paper.

\section{Materials and Methods}

Definition 1. [41,42] Fractional derivative of $f(z)$ with order $\mu$ is $f_{\mu}(z)(\mu>0)$ and fractional integral of $f(z)$ with order $-\mu$ is $f_{\mu}(z)(\mu<0)$ where

$$
\left|f_{\mu}(z)\right|<\infty \quad(\mu \in \mathbb{R}),
$$

and,

$$
\begin{gathered}
f_{\mu}(z)=\frac{\Gamma(\mu+1)}{2 \pi i} \int_{C} \frac{f(\tau) d \tau}{(\tau-z)^{\mu+1}} \quad\left(\mu \notin \mathbb{Z}^{-}, \tau \neq z\right), \\
f_{-n}(z)=\lim _{\mu \rightarrow-n} f_{\mu}(z) \quad\left(n \in \mathbb{Z}^{+}\right),
\end{gathered}
$$

where $f(z)$ is regular inside and on $C$ and,

$$
\begin{gathered}
-\pi \leq \arg (\tau-z) \leq \pi \quad \text { for } C^{-} \\
0 \leq \arg (\tau-z) \leq 2 \pi \quad \text { for } C^{+} .
\end{gathered}
$$

Definition 2. Riemann-Liouville fractional calculus definitions are, respectively [43],

$$
\begin{array}{cl}
{ }_{a} D_{z}^{-\mu} f(z)=\frac{1}{\Gamma(\mu)} \int_{a}^{z} f(\tau)(z-\tau)^{\mu-1} d \tau & (z>a, \mu>0), \\
{ }_{a} D_{z}^{\mu} f(z)=\frac{1}{\Gamma(n-\mu)}\left(\frac{d}{d z}\right)^{n} \int_{a}^{z} f(\tau)(z-\tau)^{n-\mu-1} d \tau \quad(n-1 \leq \mu<n, \quad n \in \mathbb{N}) .
\end{array}
$$

Lemma 1. (Linearity) Suppose that $f(z)$ and $g(z)$ are analytic and single-valued. When $f_{\mu}$ and $g_{\mu}$ exist, so

$$
(A f+B g)_{\mu}=A f_{\mu}+B g_{\mu}
$$

where $A$ and $B$ are constants and $\mu \in \mathbb{R}, z \in \mathbb{C}$ [29].

Lemma 2. (Index law) Suppose that $f(z)$ is an analytic and single-valued. When $\left(f_{v}\right)_{\mu}$ and $\left(f_{\mu}\right)_{v}$ exist, so

$$
\left(f_{v}\right)_{\mu}=f_{v+\mu}=\left(f_{\mu}\right)_{v^{\prime}}
$$

where $v, \mu \in \mathbb{R}$ and $z \in \mathbb{C}$ [29]. 
Lemma 3. (Generalized Leibniz rule) Suppose that $f(z)$ and $g(z)$ are analytic and single-valued. Then, generalized Leibniz rule obtained from the Sonine-Letnikov derivative is

$$
(f g)_{\mu}=\sum_{n=0}^{\infty} \frac{\Gamma(\mu+1)}{\Gamma(\mu+1-n) \Gamma(n+1)} f_{\mu-n} g_{n}
$$

where $\mu \in \mathbb{R}, \mathrm{z} \in \mathbb{C}$ and $\left|\frac{\Gamma(\mu+1)}{\Gamma(\mu+1-n) \Gamma(n+1)}\right|<\infty[30]$.

Property 1. For a constant $\lambda$,

$$
\begin{gathered}
\left(\mathrm{e}^{\lambda z}\right)_{\mu}=\lambda^{\mu} \mathrm{e}^{\lambda z}, \\
\left(\mathrm{e}^{-\lambda z}\right)_{\mu}=\mathrm{e}^{-i \pi \mu} \lambda^{\mu} \mathrm{e}^{-\lambda z}, \\
\left(z^{\lambda}\right)_{\mu}=\mathrm{e}^{-i \pi \mu} \frac{\Gamma(\mu-\lambda)}{\Gamma(-\lambda)} z^{\lambda-\mu},
\end{gathered}
$$

where $\lambda \neq 0, \mu \in \mathbb{R}, \quad z \in \mathbb{C}$ and $\left|\frac{\Gamma(\mu-\lambda)}{\Gamma(-\lambda)}\right|<\infty[30]$.

\section{Property 2.}

$$
\begin{gathered}
\Gamma(z+1)=z \Gamma(z)=z !, \\
\Gamma(\mu-n)=(-1)^{n} \frac{\Gamma(\mu) \Gamma(1-\mu)}{\Gamma(n+1-\mu)},
\end{gathered}
$$

where $n \in \mathbb{Z}_{0}^{+}$and $\mu \in \mathbb{R}$.

\section{Main Results}

In 1926, Schrödinger asserted that electrons show wave properties. Then, he defined the time-independent Schrödinger equation $H \psi=\mathcal{E} \psi$, where the wave function is $\psi$, the wave operator is $H$, and the energy of the wave is $\mathcal{E}$. In the quantum theory, the classical wave equation is

$$
\left[\nabla^{2}-\frac{1}{v^{2}} \frac{\partial^{2}}{\partial t^{2}}\right] \psi(r, \theta, \Phi, t)=0
$$

where $\nabla^{2}$ is Laplace operator, $v$ is linear velocity, $t$ is time, $r$ is radial distance, $\theta$ is polar angle and $\Phi$ is azimuth. In the CGS units, $\mathcal{V}(r)$ potential energy is

$$
\mathcal{V}(r)=-\frac{\epsilon^{2}}{r}
$$

where $\epsilon$ is the magnitude of the electron charge. When the time harmonic function is eliminated, the Schrödinger equation is

$$
\begin{gathered}
-\frac{\hbar^{2}}{2 m}\left[\frac{1}{r^{2}} \frac{\partial}{\partial r}\left(r^{2} \frac{\partial \psi}{\partial r}\right)+\frac{1}{r^{2} \sin \theta} \frac{\partial}{\partial \theta}\left(\sin \theta \frac{\partial \psi}{\partial \theta}\right)_{r, \Phi}+\frac{1}{r^{2} \sin ^{2} \theta}\left(\frac{\partial^{2} \psi}{\partial \Phi^{2}}\right)_{r, \theta}\right]+\mathcal{V}(r) \psi(r, \theta, \Phi) \\
=\mathcal{E} \psi(r, \theta, \Phi)
\end{gathered}
$$

where $\hbar$ is Planck constant $\left(\hbar=1.01 \times 10^{-34} \mathrm{Js}\right)$ and $m$ is the mass of the electron. In the spherical coordinates, the radial equation of (15) is

$$
\frac{d}{d r}\left(r^{2} \frac{d \mathcal{R}}{d r}\right)+\frac{2 m r^{2}}{\hbar^{2}}\left[\mathcal{E}-\mathcal{V}(r)-\frac{l(l+1) \hbar^{2}}{2 m r^{2}}\right] \mathcal{R}(r)=0
$$


We use the substitution $\mathcal{U}(r)=r \mathcal{R}(r)$, then we have

$$
\mathcal{U}^{\prime \prime}+\frac{2 m}{\hbar^{2}}\left[\mathcal{E}-\mathcal{V}(r)-\frac{l(l+1) \hbar^{2}}{2 m r^{2}}\right] \mathcal{U}=0
$$

and $\mathcal{U}_{\infty}^{\prime \prime}-\left(\frac{\rho}{2}\right)^{2} \mathcal{U}=0$. We obtain

$$
\rho^{2}=\frac{-8 m \mathcal{E}}{\hbar^{2}}
$$

where $\rho$ is the eigenvalue of the eigenfunction solution of the Schrödinger equation and $\mathcal{E}$ is the energy levels of the hydrogen atom [44]. In Equation (16), let $r=z / \rho$ where $\rho$ has the form of Equation (18). Then,

$$
\frac{d \mathcal{R}}{d r}=\rho \frac{d \mathcal{R}}{d z}, \quad \frac{d^{2} \mathcal{R}}{d r^{2}}=\rho^{2} \frac{d^{2} \mathcal{R}}{d z^{2}}, \quad \mathcal{V}=-\frac{\rho \epsilon^{2}}{z}, \quad \mathcal{E}=-\frac{\rho^{2} \hbar^{2}}{8 m},
$$

and,

$$
\frac{d^{2} \mathcal{R}}{d z^{2}}+\frac{2}{z} \frac{d \mathcal{R}}{d z}+\left[-\frac{1}{4}+\frac{2 m \epsilon^{2}}{\hbar^{2} \rho z}-\frac{l(l+1)}{z^{2}}\right] \mathcal{R}=0 .
$$

Let $\mathcal{R}=g / z(g=g(z))$, then we obtain at the differential equation

$$
\frac{d^{2} g}{d z^{2}}+\left[-\frac{1}{4}+\frac{2 m \epsilon^{2}}{\hbar^{2} \rho z}-\frac{l(l+1)}{z^{2}}\right] g=0
$$

For Equation (20), we use the transformation

$$
g(z)=z^{1 / 2} f(z), \quad \sigma=\frac{2 m \epsilon^{2}}{\hbar^{2} \rho}, \quad l(l+1)=\frac{\tau^{2}-1}{4} \quad(\tau=2 l+1) .
$$

Finally, we have

$$
z^{2} \frac{d^{2} f}{d z^{2}}+z \frac{d f}{d z}+\left(-\frac{z^{2}}{4}+\sigma z-\frac{\tau^{2}}{4}\right) f(z)=0
$$

Theorem 1. Let $f \in\left\{f: 0 \neq\left|f_{\mu}\right|<\infty ; \mu \in \mathbb{R}\right\}$. Equation (22) can be written as follows

$$
z^{2} f_{2}+z f_{1}+\left(-\frac{z^{2}}{4}+\sigma z-\frac{\tau^{2}}{4}\right) f=0 \quad(z \neq 0) .
$$

Equation (23) has four different fractional solutions of the forms:

$$
\begin{aligned}
& f^{(1)}=A z^{\tau / 2} \mathrm{e}^{z / 2}\left[z^{\left(\frac{-\tau+2 \sigma-1}{2}\right)} \mathrm{e}^{-z}\right]_{\frac{\tau+2 \sigma-1}{2}{ }^{\prime}} \\
& f^{(11)}=B z^{\tau / 2} \mathrm{e}^{-z / 2}\left[z^{\left(\frac{-\tau-2 \sigma-1}{2}\right)} \mathrm{e}^{z}\right]_{\frac{\tau-2 \sigma-1}{2}{ }^{\prime}} \\
& f^{(111)}=C z^{-\tau / 2} \mathrm{e}^{z / 2}\left[z^{\left(\frac{\tau+2 \sigma-1}{2}\right)} \mathrm{e}^{-z}\right]_{\frac{-\tau+2 \sigma-1}{2}}{ }^{\prime} \\
& f^{(\mathrm{iv})}=D z^{-\tau / 2} \mathrm{e}^{-z / 2}\left[z^{\left(\frac{\tau-2 \sigma-1}{2}\right)} \mathrm{e}^{z}\right]_{\frac{-\tau-2 \sigma-1}{2}},
\end{aligned}
$$

where $f_{n}=d^{n} f / d z^{n}(n=0,1,2, \ldots), f_{0}=f=f(z), \quad z \in \mathbb{C}, \tau$ and $\sigma$ are defined in Equation (21) and, $A, B, C, D$ are constants.

Proof. Let $g=g(z)$. Set

$$
f=z^{\omega} g \quad(z \neq 0)
$$


Hence, we have

$$
z g_{2}+(2 \omega+1) g_{1}+\left[\sigma-\frac{z}{4}+\left(\omega^{2}-\frac{\tau^{2}}{4}\right) z^{-1}\right] g=0
$$

Here, we choose $\omega$ such that $\omega^{2}-\left(\tau^{2} / 4\right)=0$, that is, $\omega= \pm \tau / 2$.

(I). Let $\omega=\tau / 2$. From (28) and (29), we have

$$
f=z^{\tau / 2} g
$$

and,

$$
z g_{2}+(1+\tau) g_{1}+\left(\sigma-\frac{z}{4}\right) g=0
$$

Let $h=h(z)$. Set

$$
g=\mathrm{e}^{\lambda z} h \quad(z \neq 0)
$$

and by substituting (32) into (31), we have

$$
z\left(\mathrm{e}^{\lambda z} h\right)_{2}+(1+\tau)\left(\mathrm{e}^{\lambda z} h\right)_{1}+\left(\sigma-\frac{z}{4}\right)\left(\mathrm{e}^{\lambda z} h\right)=0,
$$

hence,

$$
z h_{2}+(2 \lambda z+1+\tau) h_{1}+\left[z\left(\lambda^{2}-\frac{1}{4}\right)+\lambda(1+\tau)+\sigma\right] h=0 .
$$

In Equation (34), we choose $\lambda$ such that $\lambda^{2}-(1 / 4)=0$, that is, $\lambda= \pm 1 / 2$.

(i). Let $\lambda=1 / 2$. So, we have

$$
g=\mathrm{e}^{z / 2} h
$$

and,

$$
z h_{2}+(z+1+\tau) h_{1}+\left(\frac{1+\tau}{2}+\sigma\right) h=0 .
$$

By applying Equation (7) to both members of (36), thus the singular differential equation with integer order (36) transforms into the following fractional differential equation

$$
z h_{2+\mu}+(\mu+z+1+\tau) h_{1+\mu}+\left(\mu+\frac{1+\tau}{2}+\sigma\right) h_{\mu}=0
$$

Here, we get $\mu$ as $\mu+(1+\tau / 2)+\sigma=0$, then,

$$
\mu=(-\tau-2 \sigma-1) / 2 .
$$

By substituting (38) into (37), we have

$$
h_{\frac{-\tau-2 \sigma+1}{2}+1}+\left[\left(\frac{\tau-2 \sigma+1}{2}\right) z^{-1}+1\right] h_{\frac{-\tau-2 \sigma+1}{2}}=0 .
$$

Set

$$
h_{1+\mu}=h_{\frac{-\tau-2 \sigma+1}{2}}=u^{(1)}=u^{(1)}(z) \quad\left(h(z)=\left(u^{(1)}\right)_{\frac{\tau+2 \sigma-1}{2}}\right),
$$

and, we have then

$$
u^{(1)}+\left[\left(\frac{\tau-2 \sigma+1}{2}\right) z^{-1}+1\right] u^{(1)}=0
$$

from (39). The solution of Equation (41) is obtained by

$$
u^{(1)}=A z^{\left(\frac{-\tau+2 \sigma-1}{2}\right)} \mathrm{e}^{-z} .
$$


Then, we have Equation (24) from (34), (40) and (42). Meanwhile,

$$
h=A\left(u^{(1)}\right)_{\frac{\tau+2 \sigma-1}{2}}
$$

satisfies (39). Therefore (24) satisfies (23), since we have (30).

(ii). We get $\lambda=-1 / 2$. Then,

$$
g=\mathrm{e}^{-z / 2} h
$$

So, we obtain

$$
\begin{gathered}
\mu=(-\tau+2 \sigma-1) / 2, \\
h_{\frac{-\tau+2 \sigma+1}{2}+1}+\left[\left(\frac{\tau+2 \sigma+1}{2}\right) z^{-1}-1\right] h_{\frac{\tau+2 \sigma+1}{2}}=0, \\
h_{1+\mu}=h_{\frac{-\tau+2 \sigma+1}{2}}=u^{(11)}=u^{(11)}(z) \quad\left(h(z)=\left(u^{(11)}\right)_{\frac{\tau-2 \sigma-1}{2}}\right),
\end{gathered}
$$

with similar steps and,

$$
u^{(11)} 1+\left[\left(\frac{\tau+2 \sigma+1}{2}\right) z^{-1}-1\right] u^{(11)}=0 .
$$

The solution of Equation (48) is obtained by

$$
u^{(11)}=B z^{\left(\frac{-\tau-2 \sigma-1}{2}\right)} \mathrm{e}^{z} .
$$

Then, we have Equation (25) from (44), (47) and (49). Also,

$$
h=B\left[z^{\left(\frac{-\tau-2 \sigma-1}{2}\right)} \mathrm{e}^{z}\right]_{\frac{\tau-2 \sigma-1}{2}},
$$

satisfies (46). Therefore (25) satisfies (23), since we have (30).

(II). Let $\omega=-\tau / 2$. From (28) and (29) we have

$$
f=z^{-\tau / 2} g,
$$

and,

$$
z g_{2}+(1-\tau) g_{1}+\left(\sigma-\frac{z}{4}\right) g=0 .
$$

Let $h=h(z)$. Then, we obtain (32) and $\lambda= \pm 1 / 2$.

(iii) Let $\lambda=1 / 2$. So, we obtain (35). By applying similar steps, we have

$$
\begin{gathered}
\mu=(\tau-2 \sigma-1) / 2, \\
h_{\frac{\tau-2 \sigma+1}{2}+1}+\left[\left(\frac{-\tau-2 \sigma+1}{2}\right) z^{-1}+1\right] h_{\frac{\tau-2 \sigma+1}{2}}=0, \\
h_{1+\mu}=h_{\frac{\tau-2 \sigma+1}{2}}=u^{(111)}=u^{(111)}(z) \quad\left(h(z)=\left(u^{(111)}\right)_{\frac{-\tau+2 \sigma-1}{2}}\right),
\end{gathered}
$$

and,

$$
u^{(111)} 1+\left[\left(\frac{-\tau-2 \sigma+1}{2}\right) z^{-1}+1\right] u^{(111)}=0 .
$$

The solution of Equation (56) is obtained by

$$
u^{(111)}=C z^{\left(\frac{\tau+2 \sigma-1}{2}\right)} \mathrm{e}^{-z} .
$$


Then, we have Equation (26) from (35), (55) and (57). And,

$$
h=C\left[z^{\left(\frac{\tau+2 \sigma-1}{2}\right)} \mathrm{e}^{-z}\right]_{\frac{-\tau+2 \sigma-1}{2}}
$$

satisfies (54). Therefore (26) satisfies (23), since we have (51).

(iv) Let $\lambda=-1 / 2$. So, we obtain (44). By applying similar steps, we have

$$
\begin{gathered}
\mu=(\tau+2 \sigma-1) / 2, \\
h_{\frac{\tau+2 \sigma+1}{2}+1}+\left[\left(\frac{-\tau+2 \sigma+1}{2}\right) z^{-1}-1\right] h_{\frac{\tau+2 \sigma+1}{2}}=0, \\
h_{1+\mu}=h_{\frac{\tau+2 \sigma+1}{2}}=u^{(\mathrm{iv})}=u^{(\mathrm{vv})}(z) \quad\left(h(z)=\left(u^{(\mathrm{vv})}\right)_{\frac{-\tau-2 \sigma-1}{2}}\right),
\end{gathered}
$$

and,

$$
u^{(\mathrm{iv})}+\left[\left(\frac{-\tau+2 \sigma+1}{2}\right) z^{-1}-1\right] u^{(\mathrm{iv})}=0 .
$$

The solution of Equation (62) is obtained by

$$
u^{(\mathrm{iv})}=D z^{\left(\frac{\tau-2 \sigma-1}{2}\right)} \mathrm{e}^{z} .
$$

Then, we have Equation (27) from (44), (61) and (63). And,

$$
h=D\left[z^{\left(\frac{\tau-2 \sigma-1}{2}\right)} \mathrm{e}^{z}\right]_{\frac{-\tau-2 \sigma-1}{2}},
$$

satisfies (60). Therefore (27) satisfies (23), since we have (51).

Theorem 2. Let $\left|\left[z^{\left(\frac{-\tau+2 \sigma-1}{2}\right)}\right]_{n}\right|<\infty\left(n \in \mathbb{Z}_{0}^{+}\right), z \neq 0$ and $\left|-\frac{1}{z}\right|<1$. Equation (24) can be given as follows

$$
f(z)=z^{(2 \sigma-1) / 2} \mathrm{e}^{-z / 2} \mathbf{F}\left[1-\left(\frac{\tau+2 \sigma+1}{2}\right), \frac{\tau-2 \sigma+1}{2} ;-\frac{1}{z}\right]
$$

where $\mathbf{F}$ is the Gauss hypergeometric function.

Proof. By using (7) for (24),

$$
f(z)=A z^{\tau / 2} \mathrm{e}^{z / 2} \sum_{n=0}^{\infty} \frac{\Gamma\left(\frac{\tau+2 \sigma+1}{2}\right)}{\Gamma\left(\frac{\tau+2 \sigma+1}{2}-n\right) \Gamma(n+1)} \mathrm{e}^{-z}{ }_{\left(\frac{\tau+2 \sigma-1}{2}-n\right)}\left[z^{\left(\frac{-\tau+2 \sigma-1}{2}\right)}\right]_{n} .
$$

By using (9) and (10), we obtain

$$
\mathrm{e}^{-z}{ }_{\left(\frac{\tau+2 \sigma-1}{2}-n\right)}=\mathrm{e}^{-i \pi\left(\frac{\tau+2 \sigma-1}{2}-n\right)} \mathrm{e}^{-z},
$$

and,

$$
\left[z^{\left(\frac{-\tau+2 \sigma-1}{2}\right)}\right]_{n}=\mathrm{e}^{-i \pi n} \frac{\Gamma\left[n+\frac{\tau-2 \sigma+1}{2}\right]}{\Gamma\left[\frac{\tau-2 \sigma+1}{2}\right]} z^{\frac{-\tau+2 \sigma-1}{2}-n}
$$

where $\left|\frac{\Gamma\left[n+\frac{\tau-2 \sigma+1}{2}\right]}{\Gamma\left[\frac{\tau-2 \sigma+1}{2}\right]}\right|<\infty$. 
By substituting (67) and (68) into (66), we have

$$
f(z)=A z^{\frac{2 \sigma-1}{2}} \mathrm{e}^{\frac{-1}{2}[z+i \pi(\tau+2 \sigma-1)]} \sum_{n=0}^{\infty} \frac{\Gamma\left(\frac{\tau+2 \sigma+1}{2}\right)}{\Gamma\left(\frac{\tau+2 \sigma+1}{2}-n\right)} \frac{\Gamma\left[n+\frac{\tau-2 \sigma+1}{2}\right]}{\Gamma\left[\frac{\tau-2 \sigma+1}{2}\right]} \frac{z^{-n}}{\Gamma(n+1)} .
$$

By applying (11) and (12) to (69), then

$$
f(z)=A z^{\frac{2 \sigma-1}{2}} \mathrm{e}^{\frac{-1}{2}[z+i \pi(\tau+2 \sigma-1)]} \sum_{n=0}^{\infty} \frac{\Gamma\left[n+1-\left(\frac{\tau+2 \sigma+1}{2}\right)\right]}{\Gamma\left[1-\left(\frac{\tau+2 \sigma+1}{2}\right)\right]} \frac{\Gamma\left[n+\frac{\tau-2 \sigma+1}{2}\right]}{\Gamma\left[\frac{\tau-2 \sigma+1}{2}\right]}\left(-\frac{1}{z}\right)^{n} \frac{1}{n !} .
$$

And, we have

$$
f(z)=A z^{\frac{2 \sigma-1}{2}} \mathrm{e}^{\frac{-1}{2}[z+i \pi(\tau+2 \sigma-1)]} \sum_{n=0}^{\infty}\left[1-\left(\frac{\tau+2 \sigma+1}{2}\right)\right]_{n}\left[\frac{\tau-2 \sigma+1}{2}\right]_{n}\left(-\frac{1}{z}\right)^{n} \frac{1}{n !} .
$$

We suppose that

$$
\frac{1}{A}=\mathrm{e}^{-i \pi\left(\frac{\tau+2 \sigma-1}{2}\right)}
$$

Finally, we obtain (65) from (71).

By applying similar steps, we have Theorem $3-5$ as follows:

Theorem 3. Let $\left|\left[z^{\left(\frac{-\tau-2 \sigma-1}{2}\right)}\right]_{n}\right|<\infty\left(n \in \mathbb{Z}_{0}^{+}\right), z \neq 0$ and $\left|\frac{1}{z}\right|<1$. Equation (25) can be given as follows

$$
f(z)=z^{(-2 \sigma-1) / 2} \mathrm{e}^{z / 2} \mathbf{F}\left[1-\left(\frac{\tau-2 \sigma+1}{2}\right), \frac{\tau+2 \sigma+1}{2} ; \frac{1}{z}\right],
$$

where $\mathbf{F}$ is the Gauss hypergeometric function.

Theorem 4. Let $\left|\left[z^{\left(\frac{\tau+2 \sigma-1}{2}\right)}\right]_{n}\right|<\infty\left(n \in \mathbb{Z}_{0}^{+}\right), z \neq 0$ and $\left|-\frac{1}{z}\right|<1$. Equation (26) can be given as follows

$$
f(z)=z^{(2 \sigma-1) / 2} \mathrm{e}^{-z / 2} \mathbf{F}\left[1-\left(\frac{-\tau+2 \sigma+1}{2}\right), \frac{-\tau-2 \sigma+1}{2} ;-\frac{1}{z}\right]
$$

where $\mathbf{F}$ is the Gauss hypergeometric function.

Theorem 5. Let $\left|\left[z^{\left(\frac{\tau-2 \sigma-1}{2}\right)}\right]_{n}\right|<\infty\left(n \in \mathbb{Z}_{0}^{+}\right), z \neq 0$ and $\left|\frac{1}{z}\right|<1$. Equation (27) can be given as follows

$$
f(z)=z^{(-2 \sigma-1) / 2} \mathrm{e}^{z / 2} \mathbf{F}\left[1-\left(\frac{-\tau-2 \sigma+1}{2}\right), \frac{-\tau+2 \sigma+1}{2} ; \frac{1}{z}\right]
$$

where $\mathbf{F}$ is the Gauss hypergeometric function.

\section{Conclusions}

In this study, we applied the Sonine-Letnikov fractional derivative to the radial Schrödinger equation. We also presented fractional and hypergeometric forms of the solutions. The most important advantage of the Sonine-Letnikov fractional derivative is that it can be applied for singular equations.

Author Contributions: The authors contributed equally to this article.

Funding: This research received no external funding. 
Acknowledgments: The authors are grateful to editors and referees for valuable comments which improved the article.

Conflicts of Interest: The authors declare no conflict of interest.

\section{References}

1. Podlubny, I. Fractional Differential Equations: An Introduction to Fractional Derivatives, Fractional Differential Equations, to Methods of Their Solution and Some of Their Applications; Academic Press: San Diego, CA, USA, 1998.

2. Hristov, J. Approximate solutions to fractional subdiffusion equations. Eur. Phys. J. Spec. Top. 2011, 193, 229-243. [CrossRef]

3. Santos, M.A.F. Non-Gaussian distributions to random walk in the context of memory kernels. Fractal Fract. 2018, 2, 20. [CrossRef]

4. Hristov, J. Response functions in linear viscoelastic constitutive equations and related fractional operators. Math. Model. Nat. Phenom. 2019, 14, 305. [CrossRef]

5. Santos, M.A.F. Fractional Prabhakar derivative in diffusion equation with non-static stochastic resetting. Physics 2019, 1, 40-58. [CrossRef]

6. Sonine, N. On differentiation with an arbitrary index. Sb. Math. 1872, 6, 1-36.

7. Letnikov, A.V. On explanation of the main propositions of differentiation theory with an arbitrary index. Sb. Math. 1873, 6, 413-445.

8. Tarasov, V.E. Remark to history of fractional derivatives on complex plane: Sonine-Letnikov and Nishimoto derivatives. Fract. Differ. Calc. 2016, 6, 147-149. [CrossRef]

9. Nishimoto, K. Fractional derivative and integral, Part I. J. Coll. Eng. Ser. B 1976, 17, 11-19.

10. Nishimoto, K. Nishimoto's fractional differintegration and the solution of Legendre's differential equation. J. Coll. Eng. Ser. B 1976, 17, 21-25.

11. Nishimoto, K. Osler's cut and Nishimoto's cut. J. Coll. Eng. Ser. B 1977, 18, 9-13.

12. Nishimoto, K. Kummer's twenty-four functions and N-fractional calculus. Nonlinear Anal. 1997, 30, 1271-1282. [CrossRef]

13. Romero, S.S.; Srivastava, H.M. An application of the $\mathrm{N}$ fractional calculus operator method to a modified Whittaker equation. Appl. Math. Comput. 2000, 115, 11-21.

14. Miyakoda, T. On an almost free damping vibration equation using $\mathrm{N}$-fractional calculus. J. Comput. Appl. Math. 2002, 144, 233-240. [CrossRef]

15. Nishimoto, K. Some topics in N-fractional calculus. Res. Inst. Math. Sci. 2003, 1341, 52-76.

16. Nishimoto, K. N-fractional calculus of some multi-powers functions. Res. Inst. Math. Sci. 2008, 1579, 79-87.

17. Nishimoto, K. N-fractional calculus and its applications. , , In Proceedings of the 2nd IFAC Workshop on Fractional Differentiation and Its Applications, Porto, Portugal, 19-21 July 2006.

18. Nishimoto, K. N-fractional calculus of products of some power functions and some doubly infinite sums. Appl. Math. Comput. 2007, 187, 340-349. [CrossRef]

19. Nishimoto, K. On the N-fractional calculus of some composite functions. Res. Inst. Math. Sci. 2007, 1538, 20-29.

20. Miyakoda, T. On the N-fractional calculus of some algebraic functions. Res. Inst. Math. Sci. 2008, 1579, 50-65.

21. Nishimoto, K. N-fractional calculus of some functions which include a root sign. Res. Inst. Math. Sci. 2008, 1579, 66-78.

22. Yilmazer, R. N-fractional calculus operator $N^{\mu}$ method to a modified hydrogen atom equation. Math. Commun. 2010, 15, 489-501.

23. Miyakoda, T.; Nishimoto, K. N-fractional calculus operator method to some second order homogeneous Euler's equation. Res. Inst. Math. Sci. 2011, 1727, 22-34.

24. Nishimoto, K.; Wang, P.-Y.; Lin, S.-D. N-fractional calculus of some logarithmic functions and some identities. Res. Inst. Math. Sci. 2011, 1727, 48-59.

25. Nishimoto, K. Solutions to the homogeneous Chebyshev's equation by means of N-fractional calculus operator. Res. Inst. Math. Sci. 2011, 1772, 39-63.

26. Nishimoto, K. Solutions to the nonhomogeneous Chebyshev's equation by means of N-fractional calculus operator. Res. Inst. Math. Sci. 2011, 1772, 64-76. 
27. Yilmazer, R.; Ozturk, O. N-fractional calculus operator $N^{\eta}$ method applied to a Gegenbauer differential equation. Cankaya Univ. J. Sci. Eng. 2012, 9, 37-48.

28. Bas, E.; Yilmazer, R.; Panakhov, E. Fractional solutions of Bessel equation with N-method. Sci. World J. 2013, 2013, 685695. [CrossRef]

29. Ozturk, O.; Yilmazer, R. Solutions of the radial component of the fractional Schrödinger equation using $\mathrm{N}$-fractional calculus operator. Differ. Equ. Dyn. Syst. 2016. [CrossRef]

30. Ozturk, O.; Yilmazer, R. On applications of the fractional calculus for some singular differential equations. Progr. Fract. Differ. Appl. 2018, 4, 27-33. [CrossRef]

31. Vladimirov, V.S.; Volovich, I.V.; Zelenov, E.I. p-Adic Analysis and Mathematical Physics; World Scientific: Singapore, 1994.

32. Dong, J.; Xu, M. Some solutions to the space fractional Schrödinger equation using momentum representation method. J. Math. Phys. 2007, 48, 072105. [CrossRef]

33. Tarasov, V.E. Fractional Heisenberg equation. Phys. Lett. A 2008, 372, 2984-2988. [CrossRef]

34. Tarasov, V.E. Weyl quantization of fractional derivatives. J. Math. Phys. 2008, 49, 102112. [CrossRef]

35. Tarasov, V.E. Fractional generalization of the quantum Markovian master equation. Theor. Math. Phys. 2009, 158, 179-195. [CrossRef]

36. Tarasov, V.E. Fractional dynamics of open quantum systems. In Fractional Dynamics: Recent Advances; Klafter, J., Lim, S.C., Metzler, R., Eds.; World Scientific: Singapore, 2011; pp. 449-482.

37. Tarasov, V.E. Quantum dissipation from power-law memory. Ann. Phys. 2012, 327, 1719-1729. [CrossRef]

38. Tarasov, V.E. Fractional quantum field theory: From lattice to continuum. Adv. High Energy Phys. 2014, 2014, 957863. [CrossRef]

39. Garrappa, R.; Moret, I.; Popolizio, M. Solving the time-fractional Schrödinger equation by Krylov projection methods. J. Comput. Phys. 2015, 293, 115-134. [CrossRef]

40. Tarasov, V.E.; Tarasova, V.V. Time-dependent fractional dynamics with memory in quantum and economic physics. Ann. Phys. 2017, 383, 579-599. [CrossRef]

41. Ortigueira, M.D. Fractional Calculus for Scientists and Engineers; Springer: Berlin, Germany, 2011.

42. Campos, L.M.B.C. On the solution of some simple fractional differential equations. Int. J. Math. Math. Sci. 1990, 13, 481-496. [CrossRef]

43. Yilmazer, R.; Ozturk, O. Explicit solutions of singular differential equation by means of fractional calculus operators. Abstr. Appl. Anal. 2013, 2013, 715258. [CrossRef]

44. Mills, R.L. The hydrogen atom revisited. Int. J. Hydrog. Energy 2000, 25, 1171-1183. [CrossRef] 